\title{
Visibilidad e invisibilidad. Acerca del trabajo y las mujeres
}

\author{
Patricia Alejandra Collado \\ Universidad Nacional de Cuyo (UNCuyo), Argentina
}

\section{Visibilidad e invisibilidad. Acerca del trabajo y las mujeres}

Resumen: La crisis del empleo conjuntamente con las teorías del fin del trabajo, pusieron en discusión la centralidad del trabajo. Frente a esta situación, se observa que los debates en torno al trabajo en general (sus esferas productivas y reproductivas), su vinculación con el empleo y las formas visibles e invisibles de realizarlo han sido abordadas por una de las tradiciones más negadas del pensamiento social: el feminismo. Esta corriente intentó disputar el sentido sobre el trabajo y el no-trabajo, a propósito del incremento constante de la participación de las mujeres en el mercado laboral. Si bien los planteos feministas reconocen distintos niveles de análisis y énfasis, es necesario volver hacia su singular producción, con el objeto de desentrañar la situación de mayor explotación y opresión de los trabajadores en general y en especial de las mujeres, la que hoy se ve facilitada por la pretendida "invisibilización" o pérdida de centralidad del trabajo.

Palabras clave: mujeres, trabajo, capitalismo, precariedad laboral.

\section{Visibilidade e invisibilidade: acerca do trabalho e das mulheres}

Resumo: A crise do emprego, em conjunto com as teorias do fim do trabalho, colocou em pauta a centralidade do trabalho. Diante desta situação, considera-se que os debates em torno do trabalho, em geral (suas esferas produtivas e reprodutivas), sua relação com o emprego e as formas visíveis e invisíveis de realizá-lo, têm sido abordados por uma das tradições mais negadas do pensamento social: o feminismo. Esta corrente tentou disputar o sentido do trabalho e o não trabalho com relação ao incremento constante da participação das mulheres no mercado laboral. Embora as propostas feministas reconheçam diferentes níveis de análise e de ênfases, torna-se necessário retornar para sua singular produção, com o propósito de desvendar a situação de maior exploração e de opressão, dos trabalhadores em geral e em especial das mulheres. Situação facilitada pela pretendida "invisibilização" ou pela perda de centralidade do trabalho.

Palavras-chave: mulheres, trabalho, capitalismo, precariedade laboral.

\section{Visibility and Invisibility: the Work of Women}

Abstract: The crisis of employment, combined with theories about the end of work, place labor at the center of the agenda. Given this situation, it is found that debates around labor in general (its productive and reproductive spheres), its relation with employment and the visible and invisible forms of conducting work have been approached by one of the most neglected traditions of social thinking: feminism. Feminist thinkers have questioned the meaning of work and non-work in relation to the constant increase of the participation of women in the labor market. Although feminist proposals have different levels of analysis and emphasis, it is necessary to return to their unique production, in order to unveil the situation of greater exploitation and oppression, of workers in general and in particular of women. This situation is facilitated by the apparent "invisibilization" or by the loss of the centrality of labor.

Key words: women, labor, capitalism, precarious labor. 
El trabajo no existe para quien no lo ve. Pero sí para quien lo vive. Juan José Castillo

\section{Introducción}

En el ocaso del siglo 20 se produjo un amplio debate social y académico sobre el "trabajo" y su transformación. Esta temática tuvo repercusión, en el campo de las ideas, con un sinnúmero de posiciones que variaban desde una una perspectiva que sostiene la metamorfosis del trabajo-empleo hasta la sentencia apresurada de su final.

En este contexto de ebullición (social y teórica), algunas voces androcentradas incluyen y/o restringen la cuestión del trabajo de las mujeres como uno de los productos del proceso de degradación laboral. El trabajo femenino es postulado (junto con el de los jóvenes) como parámetro de la precariedad y vulnerabilidad en ciernes, como nuevo rostro de una pobreza "feminizada".

Sin embargo, una extensa y profunda producción feminista (teórica, conceptual, investigativa) sobre las características, designios y genealogía histórica del trabajo de las mujeres se actualiza hoy y connota una condición de ventaja epistémica para el análisis crítico del trabajo y sus sujetos/as en el capitalismo global. Su poder heurístico posibilita visibilizar lo invisibilizado, en términos de responder cómo se explota y oprime al trabajo en general y, en particular, el trabajo de las mujeres, y con ello permite considerar las condiciones que hoy asume la extorsión laboral.

Este escrito intenta retomar dicha tradición crítica a fin de pensar y analizar las condiciones de explotación del trabajo que se renuevan y/o perpetúan sobre las y los que viven de la venta de su capacidad laboral. Para analizar, entonces, las transformaciones en ciernes intentaremos articular las tradiciones críticas del marxismo y el feminismo a fin de dilucidar las condiciones y tendencias generales del trabajo en el nuevo siglo.

\section{Lo que se ve, es sólo la superficie}

Contrariamente a las posturas dominantes en los debates feministas sobre el trabajo-empleo de las mujeres a fines de los años '70, el trabajo femenino lejos de retraerse ha demostrado desde entonces una vitalidad progresiva en su crecimiento. El texto pionero de Francesca Bettio (1994) marca este punto de inflexión, desde la crisis del empleo femenino, en los años '60, hasta su revitalización. Si bien el mismo planteaba la situación particular de las trabajadoras en Italia, hoy puede ser tomado como primera aproximación a los síntomas de cambio que se estaban desarrollando en el mercado de trabajo ${ }^{1}$.
[...] la tasa de participación femenina no sólo ha continuado creciendo, sino que también ha seguido aumentando la cuota porcentual de mujeres en casi todos los sectores. Asimismo, alrededor de las tres cuartas partes del incremento neto de la ocupación global a partir de los años setenta debe atribuirse al componente femenino. Finalmente es cierto, a pesar de todo, que la desocupación femenina ha crecido paralelamente al incremento de la ocupación femenina, pero este fenómeno sugiere, más bien, que una creciente dificultad para encontrar trabajo no ha sido suficiente para frenar el aumento de la oferta femenina(BETTIO, 1994, p. 377-378).

Hasta ese momento, un conjunto de pensadoras feministas adscribían a la teoría o paradigma de la "debilidad" para explicar las restricciones que padecían las mujeres para ingresar al mercado de trabajo. Sucintamente, imputaban como impedimento para acceder al empleo problemas padecidos por restricciones de "oferta", que en el particular caso de las mujeres se conformaban en un conjunto de caracteres que se reforzaban mutuamente. Entre éstos se mencionaban como condicionantes sobresalientes la rigidez procedente de sus "obligaciones familiares" (renuencia a realizar horas extras, mayor ausentismo); las asiduas entradas y salidas del mercado de trabajo según el momento del "ciclo vital" femenino (lo cual las hacía sujetas privilegiadas de empleos a tiempo parcial); el menor nivel de instrucción o el tipo de formación adquirida en el circuito de escolarización formal (que contribuía tanto a desalentar su acceso al mercado como a preferir a varones para determinados puestos). Estos factores se esgrimían para sostener que, aún percibiendo menores salarios, las mujeres accedían en menor proporción a los empleos disponibles.

Sin embargo, la tendencia estadística al aumento del empleo femenino desde finales de los años '70 impuso la búsqueda de otro tipo de explicación, pues las debilidades de las mujeres para ingresar al mercado de trabajo no esclarecían la evidencia acerca de su crecimiento constante en el conjunto de la población económicamente activa.

Tomando siempre la perspectiva de mercado, los argumentos viraron hacia las restricciones de la "demanda". Es así que surgieron dos explicaciones: la primera daba cuenta de distintos circuitos de inserción laboral por categorías profesionales, que privilegiaban un sector primario de captación de empleo con eje en actividades realizadas por la fuerza de trabajo masculina $y$, otro sector, secundario al mismo, de actividades tradicionalmente ocupadas y ejercidas por la mano de obra femenina.

En cuanto a la segunda explicación, si bien se rastreaban las huellas en el mercado de trabajo de ocupaciones "típicas" de varones y mujeres, se hacía 
énfasis en un elemento de carácter más estructural: la división sexual del trabajo. De tal modo lo expresa Francesca Bettio, desde una mirada superadora de la restricción del empleo vista desde la oferta o de la demanda:

$\mathrm{Si}$, gracias a la supuesta rigidez de la oferta femenina, las mujeres constituyen una fuerza de trabajo secundaria, en el sentido de ofrecer prestaciones de algún modo inferiores o en todo caso menos sofisticadas ¿Cómo podría explicarse una división sistemática de las profesiones o de las tareas según sexo? Esta última revela una clara preferencia por las mujeres para determinados puestos de trabajo y por los hombres para otros; más que una gradación en la cual las mujeres ocuparían sistemáticamente el segundo lugar (BETTIO, 1994, p. 380).

Lejos de preconizar esta "preferencia" de empleos femeninos o masculinos como naturalizada, lo interesante del planteo es señalar aquello que la socialización de las mujeres pauta (cultura, tradición y religión mediante) y el mercado (el capital) acepta y profundiza: la imposición de unos perfiles y categorías profesionales a las mujeres en detrimento y/o desaliento de otros.

Lo descrito hasta aquí no pretende avalar un análisis tal como el presentado (es decir con eje en el "mercado"), sino utilizar el debate que encararon las diversas corrientes feministas por esos años (a propósito del mercado de trabajo y las diferenciaciones de género), para poner sobre la mesa un síntoma que vino a anunciar un cambio de época y a dirimir cierto estado de la discusión entonces en boga: las mujeres no constituían - ni aún hoy lo hacen - un ejército de reserva de los brazos masculinos, sino una fuerza de trabajo con singulares características las que, al cambiar el patrón de acumulación dominante, las tornaba sujetas privilegiadas de la explotación del capital.

En este sentido, dichas discusiones, hicieron visible lo invisibilizado: el cambio en el eje, desarrollo y expansión de ciertas actividades económicas.

Volvamos entonces a pensar la metamorfosis del capital haciendo énfasis en algunas de las connotaciones actuales. Asistimos a la retracción de las actividades industriales por la vía del incremento tecnológico y de la concentración y centralización del capital (ANTUNES, 1999; KATZ, 2001). Su consecuencia, en términos de mercado, es la menor cantidad de puestos de trabajo "masculinizados". Por otra parte, se produce una ampliación progresiva de actividades de servicios, circulación y comercialización, lo cual induce la creación de una mayor cantidad de puestos de trabajo, tradicionalmente "feminizados".

Esto explica la disonancia estadística referida al aumento de puestos de trabajo favorables a las mujeres, pero no nos dice nada acerca de la calidad de estos puestos y sus características. Otra de las discusiones, encaradas por el feminismo, nos da una pista sobre este nuevo eje. Los problemas asociados a emplear mujeres durante el período de acumulación industrial bajo la pauta salarial fordista fue la "estabilidad en puesto", que no es más que el anclaje del cuerpo a una determinada tarea, en el período vital completo que despliegan las personas en condiciones de acceder a la actividad laboral. Como vimos, una de las "restricciones de oferta" presentadas por las mujeres era su característica inadecuada de flexibilidad, evidente en la permanente rotación entre empleos (al interior del mercado laboral) y en la frecuencia de ciclos de actividad e inactividad (ingresos y egresos del mercado de trabajo). Esto hacía a las mujeres "desventajosas" para una disciplina laboral "permanente y fija", basamento de la producción y de la productividad del trabajo en masa.

La transformación de los modos de acumulación de capital cimentada en las "formas de organización flexible" del trabajo, nos acercan una vez más a comprender la situación de mercado: las mujeres, debido a este carácter, se presentaban como precursoras en el acceso a empleos donde la rotación y flexibilidad y, como veremos más adelante, la precariedad, es el rasgo común predominante.

Ilustraremos el escenario de los años ' 80 a través de la descripción que realiza Ricardo Antunes:

A título de ejemplo: en Francia hubo una reducción de 501 mil empleos de tiempo completo, entre 1982 y 1988; en el mismo período hubo un aumento de 111 mil empleos de tiempo parcial [...]. En 1988 [...], el 23,2\% de los asalariados de la Comunidad Europea eran empleados a tiempo parcial o en trabajos temporarios [...]. En Inglaterra, los 'trabajadores flexibles' aumentaron un 16\%, alcanzando 8,1 millones entre 1981 y 1985 [...]André Gorz agrega que aproximadamente entre un 35\% y un 50\% de la población trabajadora británica, francesa, alemana y norteamericana se encuentra desempleada o desarrollando trabajos precarios, parciales, etcétera [...]. Según Helena Hirata, el 20\% de las mujeres en el Japón, en 1980, trabajaban en tiempo parcial, en condiciones precarias (ANTUNES, 1999, p. 46).

Luego de esta pintura de la situación laboral en términos de mercado, el mismo autor concluye:

De este incremento de la fuerza de trabajo, un contingente sustancial está compuesto por mujeres, lo que caracteriza otro rasgo distintivo de las transformaciones en curso al interior de la clase trabajadora. Esta ya no es exclusivamente masculina, pero vive con un gran contingente de mujeres, 
no sólo en sectores como el textil, donde siempre hubo un gran número de trabajadoras, sino también en nuevas ramas, como la industria de la microelectrónica, para no hablar de los servicios (ANTUNES, 1999, p. 47).

Podemos decir, que el mercado laboral se "sirve" de las desigualdades de género preeminentes en detrimento de la mano de obra femenina. Y esta utilización, manifestación de un cambio estructural en la forma de acumulación del capital, es aún más extorsiva pues, para implementarse, necesita de la mutación acorde y adaptativa de las regulaciones laborales y hasta de los derechos del trabajo, que permiten que la condición de flexibilidad, otrora vista como condicionante negativo, adquiera el carácter positivo y laudatorio que asume actualmente.

Ajustemos entonces nuestra consideración en torno a esta última dimensión, referida a los derechos laborales que, como otros temas, también ha sido objeto de discusión y divisor de aguas de las posiciones entre feministas radicales y feminismo marxista.

Los derechos del trabajo, fundamentalmente aquellos de resguardo a la familia del trabajador y de aseguramiento de la misma bajo la forma de "salario familiar", fueron objeto de una crítica profunda por parte del feminismo radical, que veía en ellos por un lado, un modo de conservar y restringir a las mujeres en el ámbito de la reproducción doméstica (asegurando, al mismo tiempo, la no competitividad de las mujeres con los varones por idénticos puestos de trabajo), y por otro, la forma de mantener el control del varón sobre las actividades públicas-económicas de las mujeres (BORDERÍAS; CARRASCO; ALEMANY, 1994).

A propósito de estas posiciones ligadas a una discusión (más sustancial) sobre la articulación entre esfera de la reproducción y esfera de producción, la legislación protectora del trabajo era percibida como "regresiva" en tanto reproductora de la condición subordinada de las mujeres en el mercado de empleo y en el ámbito doméstico. Así sintetizan el estado de la cuestión Jane Humphries y Jill Rubery (1994, p. 409):

Las diferencias entre la escuela reduccionista/ funcionalista y la de la autonomía absoluta pueden ilustrarse a través de sus respectivos análisis sobre la función del 'salario familiar'. Según la escuela de la autonomía absoluta, los trabajadores luchan por el mantenimiento del salario familiar ante todo para reafirmar su posición dominante dentro de la familia. En cambio, según el enfoque reduccionista/ funcionalista, la noción de salario familiar es un mito creado por el capital - y reforzado por el movimiento sindical masculino 'corporativo'- para legitimar la explotación de las mujeres, remuneradas como si sólo trabajasen para ganar un 'salario complementario'.
Sin duda, las perspectivas enunciadas, están teñidas por un contexto socio-histórico bien diferente del que presenciamos actualmente. Podemos decir que el mismo se ubicaba en un proceso de "ampliación" de los derechos sociales y del trabajo, que permitían prever una paulatina disminución de las "desigualdades sociales" por la vía conjunta del mejoramiento de las condiciones generales de vida y del ingreso a la escolarización formal (MARSHALL; BOTTOMORE, $1998)^{2}$. Sin embargo, el derrotero seguido distó fuertemente de refrendar las previsiones más optimistas. Volviendo al punto en discusión, la prevención (sobre el objeto y fin de los derechos laborales corporativos) de las feministas radicales, no dejaba de ser atendible en el horizonte de apogeo del Estado de Bienestar, teniendo en cuenta que "El Estado otorga pero también controla, imponiendo estereotipos de conducta familiares" (PAUTASSI, 1998). Pero antes de refrendar sólo la condición opresiva del varón hacia las mujeres, se articulaba con las necesidades de la acumulación del capital, en un momento en que la "reserva de brazos" se hacía necesaria bajo el parámetro de la expansión económica y el aumento del consumo ${ }^{3}$. El lugar de la mujer, patriarcado y capitalismo mediante, podía quedar marginado a la provisión y resguardo de la reproducción biológica. Pero de allí a cuestionar las mejoras en las condiciones de trabajo (desde la unívoca concepción de la opresión patriarcal), soslayaba a estos derechos como concreciones y logros de las luchas del movimiento de los/las trabajadores en su conjunto. Como el búho de minerva (ex post facto), es más fácil comprender la situación y sus consecuencias una vez desmantelados y/o redefinidos estos derechos.

Complejizando el análisis, la re-regulación de los derechos a favor del mercado, a partir de las derrotas de los movimientos obreros y en general de las clases subalternas (desde los años '70 y '80) y la consolidación de la hegemonía neo-liberal (en los '90) impusieron una nueva disciplina laboral que legitimó la precariedad del trabajo para todos y todas. Precariedad de múltiples dimensiones que incluye, según Agulló Tomás (2000):

- la discontinuidad del trabajo (duración corta y riesgo de pérdida elevado, arbitrariedad empresarial e incertidumbre y temporalidad como norma, etc.);

- la incapacidad de control sobre el trabajo, deficiente o nula capacidad negociadora ante el mercado laboral (tanto individual como colectivamente), dependencia, autoexplotación, disponibilidad permanente y abusiva, sumisión, etc.;

- la desprotección del trabajador (pésimas condiciones laborales, sin derecho a prestaciones sociales, sin cobertura médico-sanitaria, alta discriminación, elevado índice de rotación, de explotación, segregación, etc.); 
- la baja remuneración del trabajador (salarios ínfimos, ninguna promoción ni desarrollo, formación escasa o nula, etc.).

Tornar "flexible" al conjunto de la fuerza de trabajo exigió la precarización de las relaciones y condiciones de trabajo con el fin último de asegurar una mayor productividad del trabajo por vía de la disminución de los costos laborales y la mayor extorsión de las capacidades de trabajo, a la vez que logró menguar las posibilidades de resistencia de la población que vive de la venta de su fuerza de trabajo, segregando, diferenciado y fragmentando al conjunto de los/las asalariadas ${ }^{4}$.

De tal modo que, con la transformación del mercado de trabajo fundamentalmente a partir de los años noventa, quedó en claro (entre otras cosas) que la culpabilización de las mujeres por aumentar el desempleo carecía de sostén teórico y empírico. También quedó claro que la dinámica del capital imponía a la vez el desmantelamiento de todas las "rigideces" que aumentaban los costos laborales (incluidos los derechos del trabajo y la reformulación de los Convenios Colectivos) y que, en todo caso, la flexibilidad de las mujeres resultaba conveniente para su ingreso al mercado laboral.

Hasta aquí, aquello que se ha tornado visible. Veremos ahora qué produjo tales manifestaciones de cambio en el mercado de trabajo actual.

\section{Lo que no se ve: la lógica del capital}

Retomando la cuestión de la génesis de la recomposición del capital puede ser destacado que, para salir de la crisis, el capitalismo mundial precisó de la destrucción de barreras políticas y económicas, de tal forma que se lograra remontar la alicaída tasa de ganancia, controlar la efervescencia de los conflictos socio-laborales y encontrar nuevos campos de acumulación (CHESNAIS, 2007).

Brevemente, para obtener mayores márgenes de ganancia, aumentó no sólo la productividad del trabajo, sino que además encaró una importante recomposición por vía del fagocitamento de capitales subalternos. Esto determinó la desaparición o contracción de los tejidos industriales más débiles, la mercantilización de actividades realizadas antes bajo el despliegue del aparato estatal, la succión de capitales desde la periferia hacia los centros (por la vía doble del pago de deudas y la apertura de los mercados) $y$, finalmente, por la centralización y concentración del capital bajo la égida de grandes empresas transnacionales.

Todo ello (en América Latina, específicamente) empujó a vastos sectores de la población hacia el pauperismo, que adquirió la denominación actualizada de “exclusión social." Es importante asumir que es el derrotero del capital el que determina la población necesaria y la población excedente para su reproducción. En este caso, la gran cantidad de población expulsada adoptó la connotación de "superpoblación excedente" 5 .

El inédito desempleo generado, unido a la restricción de actividades de subsistencia que ésta población pudiera realizar más allá del capital (la separación de modos y medios de subsistencia para poder pervivir), impusieron el desempleo como disciplinamiento macroeconómico masivo, padecido de conjunto por la población que vive del trabajo.

La situación planteada adquirió y adquiere un cariz mucho más intolerable para las mujeres. La debacle económico-social y fundamentalmente la redefinición de las funciones del Estado, puso a las mujeres en el lugar de "hacerse cargo de las estrategias de sobrevivencia”, responsabilizándolas y hasta haciéndolas asumir los costos sociales de la redefinición del capital y en concordancia, del desamparo producido por la retirada del Estado. En otras palabras, el desbarrancamiento de la estructura social fue puesto sobre hombros femeninos que, en la esfera doméstica, resistieron con las más diversas estrategias a la ruina del empleo y la crisis económica. A esto es lo que se ha denominado "feminización de la pobreza" que no es más que la visibilización del trabajo femenino tanto en el área pública como en la privada. En el ámbito público, en tanto mano de obra precarizada, población favorita a quien echar mano como resguardo del capital frente a los "riesgos laborales y de inversión”. En el trabajo doméstico, como jefas de hogar que desahogan, en estrategias individuales o comunitarias, el hambre de sus familias. En muchos casos, mujeres que no se resignan y toman la palabra y la acción para "visibilizar" sus demandas e impugnar la situación social imperante ${ }^{6}$. De tal modo que para las mujeres, el capitalismo de fin de siglo recrudeció sus características elementales más nocivas:

A nuestro entender la especificidad del modo de
producción capitalista reside en otros aspectos: en
las formas de subordinación de la reproducción a
la producción, en la extensión de las prácticas de
dominación de una clase al conjunto del campo
social, en la coherencia y la imbricación de las rela-
ciones sociales de clase y de sexo, en la extensión
de las luchas que desencadena el proceso de
desposesión (COMBES; HAICAULT, 1994, p. 550).

Una vez más la negación del trabajo de las mujeres tendrá su eco en las teorías sociales de fin del siglo 20, esta vez entrando a ellas bajo la minusvaloración de un trabajo aceptado como tal pero, cuya propia denominación lo menoscaba. Mayoritariamente es- 
tas actividades se desarrollan en un no-lugar: aquello que "no es" ni Estado ni es mercado, sino "tercer sector" y que hace referencia explícitamente a las actividades que, mayoritariamente, encaran las mujeres para asistir a los sectores excluidos, sosteniendo las condiciones mínimas de subsistencia a partir del trabajo voluntario femenino (RIFKIN, 1996).

Pero lo dicho hasta aquí enfatiza una de las caras de la lógica del capital, la que da cuenta de sus consecuencias hacia el mundo del trabajo que quedó inhibido del empleo asalariado ${ }^{7}$. Queda por revisar la otra faz y sus nuevas connotaciones: el mundo del trabajo asalariado.

Como hemos dicho en apartados anteriores, la pretendida invisibilidad del trabajo encubre, ni más ni menos, que nuevas formas de producción humanas transformadas en mercancías como así también intervenciones productivas de los hombres y mujeres que, en situación de trabajo, agregan más valor. Pretender que las mercancías, en la actual fase del capital, sólo toman las formas materiales y tangibles de los productos es a la vez una reducción falaz y simplificadora. Nuevas esferas de la economía quedan ancladas a la mercantilización y necesitan, del mismo modo, formas aggiornadas de circulación y colocación en el mercado, a lo que se suma cada vez más productos "intangibles": el aumento de todas aquellas actividades asociadas a los servicios, la comunicación y la producción de conocimiento. Los productos "inmateriales", creados por intervención, de la fuerza de trabajo en el proceso productivo, requieren de un conjunto de capacidades sólo comprobables en situación laboral. Competencias tales como la capacidad de comunicación, relacionamiento, entendimiento, comprensión y afecto son el piso comportamental, actitudinal e intelectual en el cual se asientan las producciones simbólicas que adquieren valor de cambio. Actitudes y aptitudes que se adquieren en la socialización y que son connotaciones atribuidas "tradicionalmente" a la fuerza de trabajo femenina.

Hasta ahora dichas características eran concebidas como un impedimento para el desarrollo de la "vida profesional" de las mujeres y se asignaban a la esfera no productiva (y por tanto no "valorada" en términos del mercado) del trabajo doméstico. Veamos la descripción del trabajo "relacional" femenino que realiza Vandelac (1994, p. 195):

En estas relaciones de donación y contradonación intervienen, como en las relaciones de mercado, bienes, servicios y sentimientos, como el orgullo, la gratificación, entre otros. Estas relaciones no tienen, sin embargo, las mismas reglas, ni la misma lógica, ni los mismos objetivos que las de la economía formal. Lo esencial no es la maximización de las satisfacciones de un individuo solitario y egoísta, sino el establecimiento y afianzamiento de los vínculos interpersonales que forman parte de la compleja estrategia de la reproducción.

Sin embargo el capitalismo en su actual fase necesita del tejido relacional que trenzan las y los trabajadores. Ante un mercado de consumo cada vez más restringido, la competencia por la colocación de una sobreproducción de mercancías adquiere los visos de una guerra furiosa entre competidores por los mismos segmentos de mercado ${ }^{8}$. Los "servicios" anclados en la captación de consumidores, la consecución de lealtades a las firmas, la aceptación de "paquetes de productos" por la vía de otros "beneficios conexos" a la adquisición de los mismos, tales como los de mantenimiento, atención al cliente o de posventa (piénsese en las cada vez mayores "oportunidades" que ofrece la telefonía celular, para tomar un ejemplo), requieren del esfuerzo personalrelacional para sostener la empresa en el mercado.

Este esfuerzo es reasignado desde la empresa a su personal quien se "autoresponsabiliza" del éxito de la misma. Para ello, los vínculos, al estilo de lo que pasa en las tramas familiares, se hacen indispensables en la gestión del producto, y demandan a la fuerza de trabajo el esfuerzo social, personal y subjetivo en su concreción.

Detengámonos a continuación, en los nuevos nichos de ganancia del sector específicamente de "servicios" asociados a la salud y a la educación. Aquí las relaciones socio-afectivas son prestaciones que adquieren valor de mercado y que requieren, como competencias "necesarias" de la fuerza de trabajo, las actitudes de contención, entrega personal, empoderamiento, asistencia y cooperación. Nada más afín a las características de la socialización de las mujeres, sólo que ahora estas actividades se realizan en la esfera "productiva", adquieren "visibilidad" y son "capitalizadas".

Aquellas capacidades de la fuerza laboral femenina que, como bien sostiene Kergoat, eran banalizadas e incluso desvalorizadas por haber sido adquiridas fuera de los canales institucionales de la cualificación, referidas a lo privado y cuya adquisición asumían como individual y no colectiva, son las competencias que hoy se reclaman al conjunto de la fuerza de trabajo (KERGOAT, 1994, p. 522). El traslado de las calificaciones (asociadas al puesto de trabajo y a las tareas desarrolladas en el mismo, pautadas en forma homogénea para igual categoría profesional) da paso a estas competencias (formas de auto-responsabilidad en el trabajo que remiten al individuo y no al puesto y que ensamblan un conjunto de capacidades puestas en acto, no certificables) ${ }^{9}$.

Estas actividades exigen a la vez, una extorsión mayor de la capacidad de trabajo, pues necesitan de la identificación del los/las trabajadoras con la em- 
presa, es decir del involucramiento con sus objetivos, adopción de sus metas como fines propios, atadura de la propia vida laboral y cotidiana al éxito y la ganancia de la empresa.

Las disciplinas laborales se "introyectan", incorporándose como dispositivos que pautan y autorregulan la conducta, de modo tal de establecer patrones de cómo se debe actuar, cuándo, cuánto y por qué.

\begin{abstract}
La disciplina descompone, fragmenta, analiza y fundamentalmente registra, abriendo un campo que comunica dos ámbitos diferenciados: el ámbito de lo perceptible con el de lo modificable; instancias estas que a su vez no pueden escindirse de un cierto objetivo a partir del cual la actividad humana deviene susceptible de ser analizada (observada, registrada y clasificada) hasta el extremo límite del más nimio de los detalles (jamás carente de importancia) (PIERBATTISTI, 2007, p. 80).
\end{abstract}

De esta forma el trabajo se torna más intenso y extenuante. Pero ¿cómo consienten los trabajadores/ as esta forma de extorsión tan implacable y erosiva de la subjetividad? ¿Cuáles son esos mecanismos que ponen como realización propia el desarrollo "libre" del otro? ¿Cuál es la gratificación que subyace a los modos que impregnan las nuevas formas de organizar y de disponer del trabajo?

La empresa estimula, suscita, emociona, premia, recompensa y también castiga, construyendo un espacio de mutuo reconocimiento en torno a objetivos impuestos; investidos libidinalmente de una energía que fácilmente descarta la unívoca explicación de la amenaza de despido como fuente de tal nivel de adhesión. De la misma forma que no hay identidad sin el Otro, frente al cual cada sujeto se determina, no hay identificación sin afecto (PIERBATTISTI, 2007, p. 93).

En la construcción de perfiles laborales acordes a los nuevos "productos" han colaborado un sinnúmero de transformaciones, que desde diferentes niveles, algunos macro-sociales y otros micro-sociales, favorecen el cambio en la composición social y el perfil de los asalariados.

Un conjunto de caracteres, que se refuerzan mutuamente, han posibilitado la construcción de una población trabajadora-asalariada cuyos destinos se atan a los designios del pathos empresario. Entre ellos puede destacarse: a) el colapso de los "colectivos de representación" del movimiento obrero y trabajador y a su forma típica de asociación sindical;

b) la cada vez más fluida segmentación de la población laboral que adopta ya los rasgos de fragmentación lisa y llana (por exacerbación de grupos cada vez más diferenciados dentro de un mismo ámbito laboral);

c) la retracción de los lugares de trabajo como espacios de visibilización de demandas; el perverso juego de "individuación" que, en las carreras profesionales, marca el paso del "compañero" de trabajo al "cliente interno";

d) la productividad mediada por la meritocracia de las carreras y aptitudes individualizadas;

e) el mismo trayecto educativo formal diferenciado y diferenciador según la clase, el sexo, cuando no la pertenencia étnica.

El resultado es, tal como en el caso del trabajo doméstico y reproductivo, el "padecimiento" del trabajo en tanto miserabilización subjetiva padecida como problema individual.

Frente a la doble connotación del trabajo productivo como explotador y opresivo, se podría objetar que los mecanismos de disciplina y control, así como también las formas novedosas de producción de mercancías no son extensivas al conjunto de la población trabajadora, sino que se concentran en las empresas de punta o reconvertidas bajo el paradigma de la "flexibilidad", limitándose asimismo a los sectores de producción de servicios. Sin embargo, no sólo en las empresas que registran una planificada modernización o reconversión se manifiestan los alcances de la reingeniería organizacional. Estas se imponen con matices y en forma desigual y combinada, al conjunto de la población bajo empleo: unas veces adoptando el formato de la reintegración de tareas por vía de la polifuncionalidad, otras por la adhesión a los sistemas de calidad o por el reencauzamiento de las capacitaciones laborales ("nuevas formas de hacer y ser en el trabajo"), o sólo restringiendo el ingreso al personal con "perfil acorde" a la "imagen de empresa"10.

Lo dicho hasta aquí puede cerrarse con una consideración que avale que la pretendida desaparirición o inmaterialidad del trabajo esconde el valor cada vez mayor del trabajo vivo. Su ocultamiento, como bien conocen las mujeres, responde a una necesidad: menoscabar el poder de aquellos que hacen el mundo con sus manos, intelecto, fuerza y creación permanente, perpetuando dicha relación de dominación y subordinación. 
Hacer visible lo invisible es solo la primer tarea que deben realizar los que pretenden construir una sociedad de hombres y mujeres libres e iguales.

\section{Palabras finales}

Se ha tratado de realizar en este escrito una asimilación de los debates pasados y actuales sobre el trabajo y sus sujetos, de tal forma de tomar como clave interpretativa, la tradición crítica que unifica marxismo y feminismo.

Esta perspectiva permitió dilucidar aquellos tópicos que para la sociología del trabajo - sobre todo desde finales de los años noventa y hasta la actualidad - se presentaban como "novedosos" y que sin embargo han sido y son parte del acervo y la producción teórica de las mujeres, cuyos esfuerzos se han centrado en visibilizar lo invisibilizado sobre el trabajo en general, las formas de valoración del mismo y las consecuencias concretas que, en su desarrollo, ha tenido para la vida cotidiana de las mujeres. Fueron las mujeres las que acertadamente pusieron en tela de juicio las fronteras resbaladizas entre trabajo productivo y reproductivo como así también entre esferas productivas e improductivas para el capital; vislumbraron los efectos $\mathrm{y}$ eficacias de los circuitos diferenciales de segmentación laboral por sexos; denunciaron el otrora inobjetable y naturalizado "doble trabajo" o "doble presencia" como cargas paralelas y alternas de la actividad femenina; pusieron en cuestión las formas diversas que asume la valoración-desvalorización del trabajo femenino para el capital; impugnaron la defensa de derechos laborales concebidos como corporativos y, hasta en algunos casos restrictivos, es decir garantizados sólo como "derechos selectivos" favorables a la porción masculina de trabajadores. ¿Cuál de los problemas enunciados no ha sido analizado $\mathrm{y}$ discutido como nudo donde se ata actualmente la suerte de los y las trabajadoras?

Todos los avances teóricos que han producido estas polémicas se dieron en el seno de un feminismo de carácter militante y político, es decir, no bajo la mera intencionalidad de alcanzar la erudición teórica sino como manera de poner aquello silenciado bajo el registro de su propia voz.

Las coordenadas que inscriben al trabajo en la actualidad como actividad que exige nuevas formas de extorsión de la potencia y creatividad de las capacidades humanas, reactualizan y re-significan la propuesta teórica-política del feminismo. Y es aquí tal vez, donde volviendo al trabajo, aparece un punto de distanciamiento final: la escisión flagrante que en el debate se observa entre aquellos que piensan y relatan la condición degradada del trabajo y quienes la viven. Queda pendiente asumir el compromiso político con el trabajo y sus hacedores y hacedoras.

\section{Referencias}

AGULLÓ TOMÁS, E. De la precariedad laboral a la exclusión social. In: CONGRESO LATINOAMERICANO DE SOCIOLOGÍA DEL TRABAJO, III, 2000. Anales... Buenos Aires. ALAST, 2000.

ANTUNES, R. ¿Adiós al trabajo? Ensayo sobre las metamorfosis y el rol central del mundo del trabajo. Buenos Aires: Colección Herramienta, Antídoto, 1999.

BETTIO, F. Segregación y debilidad. Hipótesis alternativas en el análisis del mercado de trabajo. In: BORDERÍAS, C.; CARRASCO. C.; ALEMANY, C. (Coord.). Las mujeres y el trabajo. Rupturas Conceptuales. Madrid: FUHEMICARIA. 1994. p. 371-391.

BORDERÍAS, C.; CARRASCO, C.;ALEMANY,C.(Coord.). Las mujeres y el trabajo. Rupturas conceptuales. Madrid: FUHEM-ICARIA, 1994.

BOSERUP, E. La mujer y el desarrollo económico. 1. edición en castellano. Madrid: Minerva, 1992.

COMBES, D.; HAICAULT, M. Producción y reproducción, relaciones sociales de sexo y de clase. In: BORDERÍAS, C.; CARRASCO. C.; ALEMANY, C. (Coord.). Las mujeres $y$ el trabajo. Rupturas Conceptuales. Madrid: FUHEMICARIA, 1994. p. 533-556.

CHESNAIS, F. Las contradicciones y antagonismos del capitalismo mundializado y sus amenazas a la humanidad. Revista Herramienta, Buenos Aires, n. 34, p. 7-22, marzo 2007.

HUMPHRIES, J.; RUBERY, J. La autonomía relativa de la reproducción social: su relación con el sistema de reproducción. In: BORDERÍAS, C.; CARRASCO. C.; ALEMANY, C. (Coord.). Las mujeres y el trabajo. Rupturas Conceptuales. Madrid: FUHEM-ICARIA, 1994. p. 393-423.

KATZ, C. Desequilibrios y antagonismos de la Mundialización. Revista Realidad Económica, Buenos Aires: IADE, n. 178, p. 90-121, feb.-marzo 2001.

Etapa, fase y crisis. 2002. Disponible en $<$ http:// www.filo.uba.ar/contenidos/carreras/historia>. Acceso en: Agosto 2008 .

KERGOAT, D. Por una sociología de las relaciones sociales. Del análisis crítico de las categorías dominantes a una nueva conceptualización. In: BORDERÍAS, C.; CARRASCO. C.; ALEMANY, C. (Coord.). Las mujeres y el trabajo. Rupturas Conceptuales. Madrid: FUHEMICARIA, 1994.p. 515-527

LICHTENBERGER, Y. Competencia y calificación: cambios de enfoques sobre el trabajo y nuevos contenidos de 
negociación. In: DOCUMENTOS PARA SEMINARIOS N 7. Buenos Aires: CEIL-PIETTE, 2000.

MARSHALL, T. H.; BOTTOMORE, T. Ciudadanía y clase social. Madrid: Alianza Editorial, 1998.

MARX, K. El capital. Crítica de la economía política. México, D. F.: FCE, 1968. (v. II).

PAUTASSI, L. Igualdad de derechos y desigualdad de oportunidades: ciudadanía, derechos sociales y género en América Latina. In: DOCUMENTO DE TRABAJO N. 3. Buenos Aires, FLACSO, 1998.

PIERBATTISTI, D. Mecanismos disciplinarios, dispositivos de poder y neoliberalismo: formas de intervención sobre la fuerza de trabajo. Revista Herramienta, Buenos Aires, n. 34, p. 75-98, marzo 2007.

RIFKIN, J. El fin del trabajo. Nuevas tecnologías contra puestos de trabajo: el nacimiento de una Nueva Era. Buenos Aires: Paidós, 1996.

VANDELAC, L. La economía doméstica a la salsa mercantil... o las valoraciones monetarias del trabajo doméstico. In: BORDERÍAS, C.; CARRASCO. C.; ALEMANY, C. (Coord.). Las mujeres y el trabajo. Rupturas Conceptuales. Madrid: FUHEM-ICARIA, 1994. p.152-208.

\section{Notas}

1 Es importante aclarar que la valoración de los mercados de trabajo con respecto al empleo femenino dependen de una multiplicidad de factores, entre ellos la división internacional del trabajo y el recorrido histórico de cada formación social concreta. Para dar cuenta de estos procesos en las márgenes del capitalismo central, ver el estudio de Boserup (1994).

2 Esta situación de mejora progresiva de las condiciones de vida, se circunscribe a la consideración de los países centrales.

3 Decimos "reserva de brazos" en el sentido expresado anteriormente, atendiendo al desarrollo de las actividades económicas en su conjunto, en ese momento histórico, en el marco de la división internacional y sexual del trabajo (no como reserva de brazos de actividades "masculinas").

4 Numerosos autores resaltan la capacidad política de las segmentaciones que producen los nuevos contratos laborales y su corolario, la heterogeneización de las condiciones de trabajo entre trabajadores que comparten un mismo ámbito laboral. A modo de síntesis podemos señalar aquí, sólo para Argentina, los aportes realizados al respecto porClaudia Figari, Beatriz Whele y Osvaldo Battistini, entre muchos otros investigadores del campo de estudios del trabajo.
5 De tal modo: "Son los medios del empleo y no los medios de subsistencia los que hacen ingresar al trabajador en la categoría de superpoblación. En realidad es necesario concebir esta fórmula de una manera todavía más general y vincularla a la mediación social que le permite al individuo ligarse a los medios de su reproducción y a los productos. En una palabra, se trata de las condiciones de producción y de las relaciones del individuo con ellas"(MARX, 1968, p. 109).

6 El caso de la Argentina a partir de la crisis del 2001 es paradigmático en términos de la visibilización de las mujeres como sujetas de lucha y de resistencia. Desde las experiencias urbanas que nuclean a las mismas sobre la base de organizaciones para procurarse alimento (comedores, desayunadores, huertas comunitarias, trueque), como participantes de los piquetes de desocupados y, en el ámbito rural, como luchadoras campesinas en la toma o recuperación de tierras. Sobre el tema y entre un importante grupo de autores e investigadores que han abordado la protesta y los movimientos sociales desde diferentes perspectivas se puede consultar:Almeida, Guillermo(2004); Zibecchi, Raúl(2003); Battistini, Osvaldo (2003); Svampa, Maristella y Pereyra, Sebastián,(2003); Giarraca, Norma(2001).

7 Coincidimos con autores como Ricardo Antunes (1999) o Enrique de la Garza Toledo (2003) acerca de la concepción ampliada del trabajo, más allá del empleo.

8 Como señala Katz: "La esfera del consumo constituye el primerámbito de los desajustes creados por la recomposición de la rentabilidad a expensas de los asalariados [...] Los patrones de 'confianza del consumidor' forjados durante el pleno empleo keynesiano de posguerra quedaron severamente afectados por el impacto negativo de la inestabilidad laboral sobre el nivel de ventas. Por eso, el mayor beneficio logrado por los capitalistas a través de la reducción de los costos no se ha traducido en un ensanchamiento de los mercados"(KATZ, 2002,p. 8).

9 "Ser competente es finalmente y de manera trivial saber desenvolverse en situaciones críticas, confusas, enredadas o imprevistas. Desenvolverse es tomar de los recursos de formación y experiencia para reordenarlos de manera original en función de los acontecimientos; pero esto no basta siempre, hay que poder tomar riesgos y conocer los límites, hay que poder apelar a, y movilizar redes... [...] la competencia es una relación. Una relación entre capacidades movilizadas y medios proporcionados, entre objetivos fijados y razones para actuar que cada quien define a su manera. El desarrollo de la competencia se apoya en una cultura de la interacción y del intercambio y, en materia de relaciones laborales, todo intercambio es eminentemente conflictivo" (LICHTENBERGER,2000,p. 12).

10 Dejamos de lado las transformaciones que imponen sus dispositivos de disciplina y control y que operan por fuera delámbito laboral. 
Patricia Alejandra Collado

pcollado@lab.cricyt.edu.ar

Doctora en Ciencias Políticas y Sociales por la Universidad Nacional de Cuyo(UNCuyo)

Magíster en Ciencias Sociales con Especialización en Sociología (Flacso - Argentina)

Profesora en la Facultad de Ciencias Políticas y Sociales y en Posgrado en la Maestría de Estudios Latinoamericanos de la UNCuyo

\section{UNCuy}

Maestría de Estudios Latinoamericanos

Avenida Ruiz Leal S/N

Parque Gral. San Martín

Ciudad de Mendoza - Argentina

CP: 5500 